\title{
Port 2000 - La combinaison d'un projet portuaire et d'un projet environnemental d'amorce de réhabilitation de l'Estuaire de la Seine
}

\author{
Paul SCHERRER', Pascal GALICHON ${ }^{2}$ \\ ${ }^{\prime}$ Directeur Technique du Port Autonome du Havre, Directeur du \\ projet Port 2000 \\ ${ }^{2}$ Administrateur du projet Port 2000
}

Résumé

Port 2000 consiste à développer sur un nouveau site un ensemble d'équipements portuaires destinés à accueillir et traiter les plus grands porte-conteneurs dans les meilleures conditions.

Situé à l'extrémité Nord Ouest de l'Estuaire de la Seine, cette réalisation tient compte d'un environnement estuarien qui est un lieu d'interface (fleuve-terre-mer) constitué de différents milieux qui sont complémentaires pour assurer la cohérence des chaînes alimentaires (prairies humides, roselières, vasières, bancs de sable...). Du fait de l'évolution de l'estuaire le maillon le plus faible et le plus menacé est apparu être celui constitué par les vasières nues dont l'altitude est comprise entre le niveau moyen de la mer et celui des pleines mers.

Le projet Port 2000 comprenci des mesures d'accompagnement environnemental d'un montant de 45,74 M€ (300 MF) qui s'insèrent dans un plan de gestion global de l'Estuaire, visant notamment à une véritable réhabilitation de l'Estuaire de la Seine : aménagements de vasières $(22,87 \mathrm{M} €)$, création de nouveaux reposoirs à oiseaux, renforcement de l'intérêt écologique de la réserve naturelle adjacente, création d'un espace préservé de 70 ha, création d'un observatoire de la pêche.

\section{Abstract}

Port 2000 consists in developing a new site with harbour facilities designed to receive the largest container vessels under optimum conditions.

Situated on the extreme north-west tip of the Seine estuary, the works take into account an estuarine environment forming an habitat which is interfacial in nature (involving river, land and marine environments), enabling the simultaneous existence of a wide range of complementary biotopes (sand banks, mudflats either bare or colonized by plants, marshes and wetlands), thereby ensuring the cohesion of the food chain. As a result of the evolution of the estuary, the most fragile and most threatened link in the food chain appears to be that formed by the bare mudflats, found at a level between the average sea level and the high tide level.

The Port 2000 project includes supportive environmental measures (45,74 M€ i.e. $300 \mathrm{MF}$ ) included themselves in a global management plan for the whole of Seine Estuary with notably the starting of a true rehabilitation over the estuary: Mudflat development $(22,87 \mathrm{M} €)$, ne' $\mathrm{N}$ resting areas for sea birds, improvement of the ecological interest of the nearby nature reserve, creation of a 70 ha preserved area, creation of a fishing activities observatory. 


\section{Le projet portuaire Port 2000}

\subsection{Objet et contexte portuaire du projet}

Le projet Port 2000 consiste à développer sur un nouveau site un ensemble d'équipements portuaires destinés à accueillir et traiter les plus grands porte-conteneurs dans les meilleures conditions permettant de fixer au Havre les escales des grandes lignes maritimes conteneurisées. Il vise à améliorer également les transferts entre les navires et les modes de transport d'approche de masse que sont les trains, les caboteurs maritimes et les barges fluviales. Il doit ainsi contribuer à améliorer la position de la France dans la logistique européenne, tout en participant au maintien des fonctionnalités biologiques de l'Estuaire de Seine.

Compte tenu de l'importance des emplois portuaires pour la région du Havre ( $30 \%$ environ des emplois dans une zone où le taux de chômage dépasse $17 \%$ ), le projet Port 2000 est d'un intérêt majeur pour Le Havre. Mais l'enjeu socio-économique de Port 2000 est bien plus vaste encore et s'étend non seulement à l'Estuaire de la Seine au sens large mais aussi à la région normande et même la France avec, en particulier, la volonté de développer une logistique-distribution à destination de l'ensemble de l'Europe.

Par ailleurs, ce projet est fondamental en termes d'aménagement du territoire français par la volonté d'augmenter très fortement les parts modales du fluvial et du fer ainsi d'ailleurs que du cabotage intra-européen. Il s'agit grâce à Port 2000 de privilégier les axes Est-Ouest sur les axes Nord-Sud de plus en plus saturés, voire au bord de l'asphyxie.

\subsection{Le Débat Public sur le projet Port 2000}

En application de la loi Barnier du 2 février 1995, un Débat Public, le premier en France, sur le projet Port 2000 s'est déroulé dans toute la Normandie et à Paris du 24 novembre 1997 au 23 mars 1998.

En fin de débat, le Port Autonome a été en mesure de faire évoluer sensiblement ses projets initiaux et proposer une solution de long terme comprenant un investissement à l'extérieur du port actuel avec potentiellement des aménagements intérieurs et des travaux de génie écologique destinés à préserver voire développer les fonctionnalités biologiques de l'estuaire qui sont actuellement en régression.

\subsection{Le projet Port 2000 pris en considération par le Gouvernement français}

Dès après la publication du compte-rendu officiel du Débat Public et du bilan établi par M. le Président de la Commission Nationale du Débat Public le 17 juin 1998, le Conseil d'Administration du Port Autonome du Havre a voté à l'unanimité le 3 juillet 1998 le projet Port 2000 sous forme d'un schéma de développement du Port du Havre et de contribution à la gestion de l'Estuaire de la Seine. Ce schéma a été adressé avec tous les éléments socio-économiques, 
techniques et financiers au Ministère des Transports le 24 juillet 1998 qui l'a pris en considération le 5 décembre 1998 y compris les aménagements visant à contribuer au maintien de la biodiversité de l'estuaire et de nouvelles dessertes proches des nouveaux terminaux. Le coût global de la part actuellement financée de ce schéma (4 postes extérieurs en $1^{\text {ère }}$ phase suivis de 2 autres postes extérieurs) est de $3724 \mathrm{MF}$ (568 M€) y compris les mesures environnementales évaluées à 300 millions de FF (45,74 M€). A cette somme, il convient d'ajouter environ 1,5 milliard de $\mathrm{F}$ (228 M€) d'investissements privés.

\subsubsection{Nouvelles installations portuaires}

La définition d'un nouveau plan masse à l'issue du Débat Public a permis une meilleure utilisation de l'espace situé à l'intérieur des nouvelles digues de délimitation (voir carte jointe).

Cette nouvelle installation se caractérisera principalement par un long quai rectiligne d'un potentiel global de 4200 mètres de long représentant douze postes à quai dont les terre-pleins, larges d'environ 500 mètres, seront localisés au Sud du dépôt pétrolier de la Compagnie Industrielle Maritime (CIM). Les 1700 mètres les plus à l'Est de ce quai sont réalisés dans des terrains actuellement remblayés.

Pour accéder à ce quai, un chenal sera creusé à la cote marine de $(-16 \mathrm{~m})$. Il rejoindra le chenal actuel à un peu plus d'un kilomètre à l'Ouest de la passe d'entrée actuelle. Le chenal aura une largeur finale de 350 mètres. Pour permettre la manœuvre des navires, un cercle d'évitage de $700 \mathrm{~m}$ de diamètre est envisagé vers le milieu du quai, ce cercle d'évitage empiétant légèrement (environ 30 hectares) sur la Zone de Protection Spéciale pour les oiseaux.

Pour protéger des houles et des courants l'ensemble du quai, des digues d'une longueur cumulée de l'ordre d'environ 5000 mètres seront construites avec une passe d'entrée délimitée par deux caissons-musoirs.

\subsubsection{Nouvelles dessertes terrestres des nouveaux terminaux}

L'augmentation attendue du trafic sur chaque mode terrestre, même si la répartition modale est appelée à se modifier en faveur du mode ferroviaire et du mode fluvial, impose de développer pour les trois modes des aménagements performants en termes de capacités et de compétitivité pour l'accès et la desserte des terminaux par des axes situés au Sud, le long de l'estuaire, permettant ainsi une séparation des trafics portuaires et urbains.

- pour le ferroviaire : la réalisation d'infrastructures ferroviaires proches des terminaux est indispensable pour permettre la constitution de trains complets pouvant directement rejoindre le réseau ferré national. Ceci pourra, selon la taille des terminaux à conteneurs et les souhaits de leurs exploitants, être réalisé soit par des voies ferrées allant directement sur les terminaux, soit par des chantiers 
ferroviaires communs à plusieurs terminaux desservis par des ensembles multi-remorques,

- pour le fluvial : il est prévu de réaliser dans la Darse de l'Océan (donc en amont de l'Écluse François $1^{\mathrm{er}}$ ) un terminal dédié fluvial desservi par des ensembles multi-remorques en site propre depuis les différents terminaux à conteneurs. Les ensembles fluviaux viendront donc escaler à ce terminal dédié à leur trafic pour $y$ embarquer ou débarquer sans délai les conteneurs qui vont ou proviennént de divers terminaux maritimes,

- pour le transport routier : l'amélioration des dessertes routières comprend notamment la restructuration du réseau local du réseau routier pour l'ensemble des terminaux du sud de la zone portuaire (aménagement de postes de contrôles, séparation des différents flux...) et le renforcement de la Route de l'Estuaire jusqu'au Pont de Normandie.

L'ensemble a fait l'objet d'une étude paysagère globale pour garantir une bonne insertion et une bonne unité du projet.

\section{Le contexte environnemental du projet}

Situé à l'extrémité Nord Ouest de l'Estuaire de la Seine, Port 2000 doit tenir compte d'un environnement estuarien qui est un milieu d'interface (fleuve-terre-mer). De nombreux facteurs interviennent, ce qui permet l'existence de milieux divers et complémentaires (bancs de sable, vasières nues ou colonisées par des végétaux, prairies et zones humides) présentant une biodiversité intéressante.

Paramètre essentiel dans l'évolution de milieux, l'hydrodynamique de l'Estuaire de la Seine est régie par le débit de la Seine qui a une valeur moyenne de $450 \mathrm{~m}^{3}$ par seconde et surtout par la marée, avec les variations de niveaux d'eau et de courants qu'elle induit. Ses caractéristiques majeures sont d'une part, son marnage ( $8,00 \mathrm{~m}$ par grandes marées) et d'autre part, le fait que les courants liés à la marée montante sont beaucoup plus forts que ceux observés à marée descendante.

La dissymétrie des courants de marée et l'action des houles favorisent le comblement de l'estuaire par des sédiments (sables et vases) qui sont essentiellement d'origine marine. Ces apports séculaires de sédiments ont varié au cours du temps, ainsi entre la fin du XVII ${ }^{\mathrm{eme}}$ siècle et les années 1990 les surfaces soumises au balancement des marées sont passées de $140 \mathrm{~km}^{2}$ à $30 \mathrm{~km}^{2}$. Les apports sont estimés aujourd'hui à environ 3 millions de $\mathrm{m}^{3}$ par an (ceux d'origine fluviale sont de l'ordre de 0,6 million de $\mathrm{m}^{3}$ par an en moyenne). Ils ont pour effet principal de déplacer l'ensemble de l'embouchure de l'estuaire vers l'aval à une vitesse qui est estimée à 50 mètres par an pour les vingt dernières années ainsi que de provoquer un comblement des vasières soumises au balancement de la marée qui actuellement ne subsistent plus que de part et d'autre du Pont de Normandie. En effet, lors de sa progression vers l'Ouest, l'estuaire se développe dans des zones 
où les énergies liées aux houles et aux courants sont plus importantes et de ce fait les vases qui ont besoin de milieux calmes pour pouvoir se déposer de manière pérenne ne peuvent le faire. La surface résiduelle de cette vasière n'est ainsi plus que de 300 ha environ et se réduit d'environ 25 ha par an.

Les nombreuses études environnementales faites sur l'Estuaire de la Seine, l'analyse de ses fonctionnalités biologiques ont mis en évidence que l'estuaire était constitué de différents milieux qui sont complémentaires pour assurer la cohérence des chaînes alimentaires (prairies humides, roselières, vasières, bancs de sable...). Du fait de l'évolution de l'estuaire le maillon le plus faible et le plus menacé est apparu être celui constitué par les vasières nues dont l'altitude est comprise entre le niveau moyen de la mer et celui des pleines mer (la haute slikke). Ce secteur qui est en régression d'une vingtaine d'hectares par an, présente en effet une productivité biologique importante. Les fonctionnalités de ces vasières tiennent principalement dans les rôles privilégiés qu'elles assurent pour :

- l'accueil des plus jeunes poissons car elles constituent un maillon essentiel du système composite qu'est une nourricerie de poissons,

- certaines espèces d'oiseaux pour lesquels l'Estuaire de la Seine revêt une importance nationale ou internationale car les vasières sont des zones où elles trouvent de quoi se nourrir et se reposer,

- le stockage et la transformation d'une part du flux des contaminants venant de l'amont de la Seine, sans qu'aucune quantification de cette fonction ne soit, à ce jour, possible.

Ainsi, il apparaît que l'enjeu environnemental majeur de l'Estuaire de la Seine se situe principalement dans le maintien de ces fonctionnalités liées aux vasières soumises aux balancements de la marée, vasières qui sont incontestablement le maillon le plus faible de l'estuaire et qu'il convient d'intégrer ce point dans la conception du projet.

Le Débat Public a fait apparaître l'intérêt de profiter de l'opération portuaire Port 2000 pour y associer une opération de génie écologique visant soit à créer de nouvelles vasières intertidales stables, soit à remettre en vasière des zones actuellement devenues des prairies humides ou des roselières, soit également à retarder l'assèchement naturel et inexorable des vasières intertidales actuelles.

Une autre fonctionnalité importante de l'estuaire sera affectée par le projet. Dans une ancienne chambre de dépôt de dragages non totalement remplie dans les années 70, une zone d'eau peu profonde d'environ 35 ha est devenue un important reposoir à oiseaux, accueillant en hiver jusqu'à 8000 oiseaux de 65 espèces différentes. La France a désigné cette zone comme ZPS (Zone de Protection Spéciale pour les oiseaux) auprès de l'Union Européenne. La disparition de ce reposoir qui sera redragué partiellement dans le cadre de la création du nouveau 
bassin de Port 2000, nécessite en conséquence la création de nouveaux reposoirs à oiseaux.

\section{Princinales mesures environnementales liées à Port 2000}

Dans sa décision de prise en considération de Port 2000, le Ministre de l'Équipement, des Transports et du Logement a demandé qu'une synthèse de l'ensemble des études sur l'Estuaire permettant de préciser les modalités d'évolution des milieux naturels soit réalisée par un Comité d'Experts indépendants présidé par M. le Préfet de Région Haute-Normandie. A partir de celle-ci, le comité a établi les axes d'un plan de gestion de l'Estuaire allant bien au-delà de Port 2000 ainsi que des propositions d'actions et de travaux écologiques à entreprendre dans le cadre du projet portuaire.

Le projet Port 2000 n'est donc qu'une partie de ce plan de gestion globale. Les mesures d'accompagnement environnemental de Port 2000 ont été arrêtées sur la base des conclusions du Comité d'experts. Elles représentent un montant global de $300 \mathrm{MF}(45,75 \mathrm{M} €)$.

Les mesures les plus importantes vont concerner les vasières intertidales avec un programme de quelques $150 \mathrm{MF}$ en vue, comme l'ont souligné les experts, d'amorcer une véritable réhabilitation de l'Estuaire de la Seine.

\subsection{La réhabilitation des vasières}

\subsubsection{Définition des objectifs recherchés}

Les nombreuses rencontres qui ont eu lieu entre des scientifiques s'intéressant à l'estuaire, les différents utilisateurs de cet espace (ports, pêcheurs, chasseurs, ornithologues, biologistes, environnementalistes...) et les administrations ont permis de définir des objectifs en ce qui concerne la création ou la réhabilitation de vasières.

a) Produire en abondance du benthos accessible aux oiseaux et aux poissons comme source d'alimentation

L'analyse des chaînes alimentaires des oiseaux limicoles et des juvéniles de poissons dans l'estuaire de la Seine a montré qu'un zoobenthos essentiellement constitué par du Corophium volutator, Hediste diversicolor et Macoma balthica constituait un compromis entre abondance, diversité (taille et comportement des proies) et accessibilité. Ce type de milieu est caractéristique des niveaux moyens et hauts des vasières intertidales.

\section{b) Assurer la pérennité dans le temps de l'aménagement}

Une vingtaine d'hectares de vasières disparaisse chaque année principalement par progression des herbus, les nouveaux aménagements devront pouvoir évoluer à un 
rythme beaucoup plus lent. L'analyse des profils d'équilibre des vasières dans l'Estuaire de la Seine montre que certains sont plus stables que d'autres en fonction des facteurs hydrodynamiques présents et qu'il conviendra d'approcher dès la réalisation ces profils pour diminuer les coûts de maintenance de l'aménagement.

\section{c) Prendre en compte la fonctionnalité écologique}

Les vasières constituent un maillon d'un ensemble de milieux et il faut que les oiseaux et les poissons puissent se déplacer aisément d'un milieu à l'autre. Il faut donc que, par exemple, les reposoirs à oiseaux à marée haute ne soient pas trop éloignés de la future vasière ou que pour les poissons juvéniles une continuité des milieux soit assurée avec des lieux de nourriceries vers lesquels ils migreront au cours de leur croissance.

d) Assurer la cohérence de gestion avec les autres milieux

Dans l'Estuaire de la Seine existent une Réserve Naturelle et un Parc Régional. La gestion environnementale des autres zones de l'estuaire ne peut donc pas se faire sans tenir compte de ces éléments et doit être définie en accord avec les gestionnaires de ces espaces.

\subsubsection{Définition d'un schéma d'aménagement}

L'examen des critères exposés précédemment a conduit à l'analyse de la potentialité des sites les plus favorables pour la création de vasières intertidales. La confrontation des avis des différents experts a conduit à travailler sur le secteur se situant de part et d'autre du Pont de Normandie.

Les études basées sur des modélisations numériques et physiques et sur les avis d'experts ont conduit à retenir le schéma présenté figure 3.

L'aménagement envisagé comporte principalement :

- une nouvelle brèche de $550 \mathrm{~m}$ de large arasée à $+2,50 \mathrm{~m} \mathrm{CMH}$ au droit de la vasière artificielle située en amont du Pont de Normandie,

- un rehaussement de la brèche actuelle à $+3,50 \mathrm{CMH}$ et de $1 \mathrm{~m}$ de la digue Basse Nord sur 2,5 km à l'aval de la brèche actuelle,

- un chenal courbe reliant la nouvelle brèche au chenal de la fosse Nord se situant au droit de la brèche actuelle. Environ $1,5 \mathrm{Mm}^{3}$ sont à draguer. Ces matériaux seront mis en dépôt de part et d'autre de la brèche actuelle pour y renforcer les bancs sablo-vaseux qui y sont, - un épi de $550 \mathrm{~m}$ situé à $2,5 \mathrm{~km}$ à l'aval de la brèche actuelle pour permettre le développement du banc de la passe. 


\subsection{Nouveaux reposoirs à oiseaux}

Pour compenser la disparition du reposoir actuel, quatre gabions de chasse à l'Ouest de la vasière actuelle ont été transformés en un grand reposoir (environ $40 \mathrm{ha}$ ) avec en son centre une île favorable à la nidification des oiseaux.

Il est également prévu de construire un îlot artificiel dans l'embouchure de la Seine, au Sud de la digue basse Sud de calibrage du chenal du Port de Rouen. Cet îlot ainsi que le montre l'expérience d'îles naturelles ou artificielles existant en d'autres points du globe sera particulièrement favorable à l'accueil des oiseaux marins et éventuellement à des mammifères marins.

\subsection{Renforcement de l'intérêt écologique de la réserve naturelle}

Port 2000 contribuera à l'amélioration de la qualité environnementale de la Réserve Naturelle de l'Estuaire de la Seine (créée le $1^{\text {er }}$ janvier 1999) grâce à de l'ingénierie écologique comportant en particulier des travaux hydrauliques et des fonds en faveur de la chasse et de l'agriculture destinés à faciliter une adaptation des pratiques de ces activités pour les rendre plus compatible avec la Réserve Naturelle.

Tout sera fait pour que la Réserve Naturelle soit la plus attractive possible pour les oiseaux cherchant à y nicher ou à s'y reposer au cours de leurs migrations entre le Nord de l'Europe et l'Afrique.

\subsection{Création d'un espace préservé de 70 ha}

$\mathrm{Au}$ Sud immédiat du projet, différentes espèces d'orchidées protégées, dont la Liparis de Loesel, ont été identifiées au cours des inventaires floristiques. En conséquence, le projet a été modifié en décalant sensiblement vers le Nord les accès terrestres. Ceci va permettre la création de 70 ha d'espace préservé non seulement pour les orchidées mais également pour les nombreux amphibiens de la zone et également les oiseaux. Cet espace fera l'objet d'un classement en réserve naturelle volontaire.

\subsection{Création d'un observatoire de la pêche}

Face à la lacune de données disponibles concernant la pêche, il est mis en place sous l'autorité du Préfet et avec une contribution forte du PAH un observatoire de la pêche qui a pour objectif, d'une part, de recueillir des données sur la socio-économie de la pêche et, d'autre part, d'évaluer aussi bien sur le court terme que sur le long terme (10 ans), l'impact du projet Port 2000 sur les ressources halieutiques et leur exploitation. Les pêcheurs et des scientifiques sont parties prenantes dans cette démarche. 


\section{Le suivi environnemental du projet Port 2000}

Le paragraphe précédent ne présente que les mesures environnementales liées à Port 2000 les plus significatives. Elles font partie d'un ensemble cohérent fondé sur les conclusions du Comité d'Experts indépendants décidé dès la prise en considération ministérielle.

Ces différentes réalisations feront l'objet de programme de suivi pour évaluer leur productivité aussi bien en terme de diversité qu'en terme de densité des espèces rencontrées.

Les arrêtés interpréfectoraux du 23 octobre 2000 portant autorisation de réaliser les installations, ouvrages et travaux prévus dans le cadre de l'extension des infrastructures portuaires dit "Port 2000", précisent que : "les modalités de suivi scientifique seront définies par le Préfet de Seine-Maritime après avis du Conseil Scientifique et Technique".

Ce Conseil Scientifique et Technique a été instauré par M. le Préfet de Haute-Normandie dans le cadre de la mise en place du Comité de Gestion Globale de l'Estuaire de la Seine à la demande de Mme le Ministre de l'Aménagement du Territoire et de l'Environnement et M. le Ministre de l'Équipement, des Transports et du Logement. Il est composé de 15 membres représentant les différents domaines scientifiques relatifs à l'écologie estuarienne. Il a notamment en charge le pilotage du suivi environnemental de Port 2000 dans la durée et il doit en assurer la cohérence par rapport aux autres programmes scientifiques dont l'Estuaire de la Seine fait l'objet (notamment le programme Seine-Aval, les études dans le cadre du Plan de Gestion de la Réserve Naturelle).

Il est à noter que les suivis pouvant présenter un caractère scientifique sont de deux types, d'une part, ceux qui relèvent de l'auto-surveillance telle que définie dans l'arrêté du 23 octobre 2000 et qui sont liés à la période du chantier (il s'agit essentiellement de contrôle de la qualité des eaux et des sédiments dans les zones affectées par les dragages) et, d'autre part ceux qui constituent des suivis scientifiques une fois les ouvrages portuaires ou environnementaux réalisés.

Ces suivis environnementaux feront l'objet de documents de synthèse semestriels qui seront régulièrement présentés au Conseil Scientifique et Technique, au Conseil de 1'Estuaire et au Comité de suivi qui, dans le cadre du Plan de Gestion Global de l'Estuaire, regroupe l'ensemble des acteurs de l'Estuaire (Élus, associations, organismes professionnels...). Ainsi à travers ces différentes instances, les résultats de ces suivis seront largement diffusés et portés à la connaissance du public. 


\section{CONCLUSION}

L'opération Port 2000 est désormais bien engagée avec en particulier :

- au printemps 2001, le transfert hors zone de chantier de plus de 2500 batraciens (espèces protégées),

- la réalisation à l'automne 2001 du nouveau reposoir sur dune,

- le lancement des chantiers "Digues et dragages associés" (Groupement GTM Dredging International), "Quais et création de darse associée" (Entreprise SOLÉTANCHE BACHY).

Outre les Plans d'Assurance Qualité désormais classiques, les divers chantiers font l'objet du Plan d'Assurance Environnementaux et sont assurés par des entreprises qui sont, pour la plupart, certifiés ISO 14001.

Ainsi, dès 2004, la France sera dotée d'un nouvel outil portuaire performant dans la compétition très sévère des ports à conteneurs du Nord de l'Europe et un important plan de réhabilitation de l'Estuaire de la Seine aura été amorcé, avec une volonté clairement affichée à tous les niveaux d'une gestion globale et équilibrée, environnementale, sociale, économique (en un mot durable) de cet estuaire.

liste des illustrations :

1. Photomontage de l'ensemble du projet

2. Schéma du projet dans ses diverses composantes

3. Schéma de l'aménagement de vasières de part et d'autre du Pont de Normandie

\section{Références :}

- compte rendu de la Commission Particulière du Débat Public Port 2000 (juillet 1998) incluant le dossier PAH pour le Débat public (115 pages et 220 pages d'annexes comptes rendus de réunions),

- résumé non technique de la première phase de l'étude d'impact (janvier 2000 , 100 pages format $\mathrm{A} 3$ ),

- étude d'impact de la première phase de Port 2000 (janvier 2000, 480 pages

+ volume d'annexes graphiques A3). 
VIrèmes Journées Nationales Génie Civil-Génie Côtier, Anglet, France, 15-17 Mai 2002
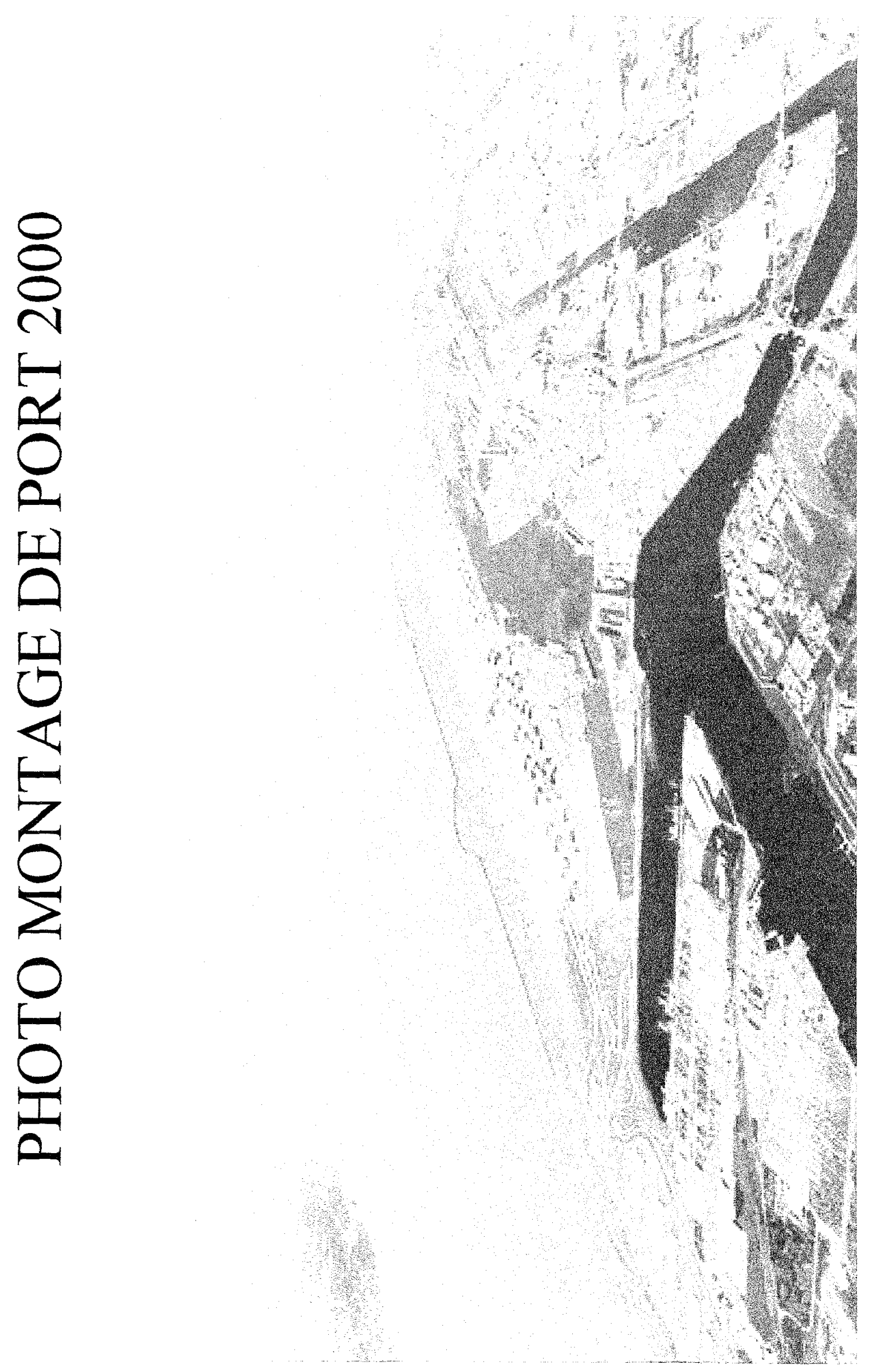







\section{SCHEMA DES AMENAGEMENTS EN VUE DE LA REHABILITATION DES VASIERES}

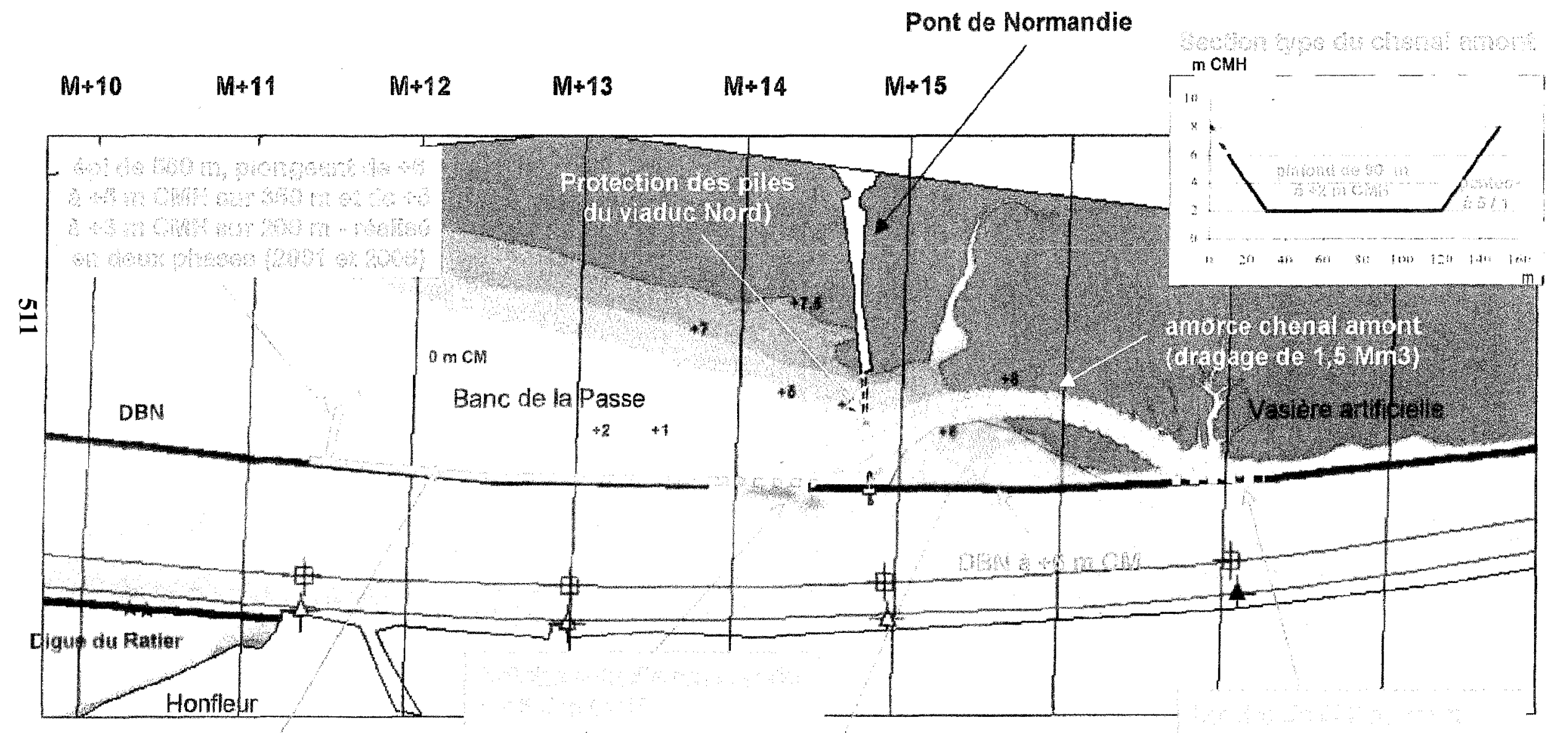

